\title{
Response to Selection and Associated Changes in Genetic Variance for Soluble Solids and Titratable Acids Contents in Strawberries
}

\author{
Douglas V. Shaw \\ Department of Pomology, University of California, Davis, CA 95616 \\ "Additional index words. Fragaria $\times$ ananassa, flavor components, heritability, selection response
}

\begin{abstract}
Parental potential, or breeding value, was evaluated for strawberry (Fragaria $\times$ ananassa Duch.) genotypes selected for high, intermediate, or low phenotypic expression of soluble solids content (SSC) and titratable acid content (TA). Progeny means and genetic variance parameters were estimated using seedlings from 40 crosses among these selections, conducted in two factorial sets. Selection response for breeding value was detected for SSC in the upward direction and for TA in both upward and downward directions. Populations created by divergent selection of parents followed by intermating expressed additive genetic variances 2.3 and 9.3 times larger than those estimated for appropriate controls for SSC and TA, respectively. For TA, the response to selection for breeding value was consistent with results for genotypic selection reported earlier. The significant selection response in breeding value obtained for SSC differed from the results of previous clonal tests that had detected no significant genotypic selection response. These results, together with previous assessments, suggest that cumulative genetic gain can be obtained for both SSC and TA. However, gain for SSC will be contingent on selection under conditions that approximate commercial treatment; this will not be necessary for TA, as expression for this trait is stable across test location and cultural treatments.
\end{abstract}

Strawberry flavor is conditioned in part by the balance between SSC and TA expressed in ripe fruit (Sweeney et al., 1970). Genetic variation for SSC and TA in strawberry can be exploited by selection and propagation of 'specific genotypes and by choice of appropriate' parents and control of cross combinations. When genetic effects are large and mostly additive, both genotypic values and breeding values are predictable from the performance of individual seedling phenotypes. However, if dominance effects are large, the correspondence between genotypic value and breeding value will be low and clonal performance may be a poor predictor of parental value. The effectiveness of mass selection in detecting superior parents and genotypes for SSC and TA depends on the mode of inheritance for genes that condition these traits.

The study reported here is the third "in a series aimed at understanding the inheritance of SSC and TA in strawberries and deriving selection strategies for developing cultivars with improved flavor. Broad-sense heritabilities $\left(\mathrm{H}^{*}\right)$ and narrow-sense heritabilities $\left(\mathrm{h}^{*}\right)$ were estimated in an earlier study, using seedlings from controlled crosses among genotypes representative of current advanced-generation germplasm (Shaw et aI., 1987). Results from this initial genetic survey demonstrated large dominance effects for SSC $\left(\mathrm{h}^{2}=0.07\right.$ and $\left.\mathrm{H}^{2}=0.35\right)$. Both additive and dominance effects were important for TA $\left(\mathrm{h}^{*}=0.48\right.$ and $\mathrm{H}^{*}=0.78$ ). Bidirectional (divergent) selection was performed for SSC and TA arrmng the seedlings evaluated in this initial survey, and selected gc rmtypes were tested using runner plan\% in clonal trials (Shaw, 1988). Results from these clonal tests demonstrated genotypic selection response for TA but not for SSC. Both the propagation system (seedling vs. runner) and the test location (inland vs. coastal) differed between the original seedling survey and subsequent clonal tests, with the clonal tests intended to simulate commercial conditions. The failure to obtain genotypic selection response for SSC couId reflect in-

Rcceived for publication 19 Dec. 1989. The cost of publishing this paper was defrayed in part by the payment of page charges. Under postal regulations, this paper therefore must be hereby markedadvertisementsolely to indicate this fact. accuracy or imprecision of the original genetic parameter estimates or might result from interaction of genotypes with propagation systems or test locations. The practical consequence here is that genotypes selected for specific levels of acidity as seedlings will express corresponding levels of acidity when propagated clonally. Conversely, seedlings selected for specific SSC phenotypes will not reliably express these values when propagated commercially.

The objectives of the following study were to: 1) evaluate response to mass selection for parental potential, or breeding value, for a subset of the genotypes included in the clonal tests described above (Shaw, 1988); 2) compare selection response for breeding value with the response demonstrated previously for genotypic selection; and 3) quantify the changes in genetic variance that result from divergent selection followed by intermating.

\section{Materials and Methods}

Seedlings from 40 biparental crosses were established in field trials on 14 Sept. 1987 at the Wolfskill Experimental Orchard, near Davis, Calif., and cultured as described by Shaw et al. (1987). Two sets of 20 crosses were evaluated, each resulting from matings among nine parental genotypes. Parents for Set I included three each selected for high $(\mathrm{H})$, intermediate (I), or low (L) phenotypic expression of SSC, whereas parents for Set II included two, three, and four items selected for high, intermediate, and low TA, respectively. Parents for Set II were a subsample of the genotypes tested by Shaw (1988) and were chosen at random within the three classes. Genotypes used as SSC parents for Set I were also a subsample of those tested previously, but selections representing high and low SSC were chosen only from crosses in the top and bottom $20 \%$ (five of the original 28 crosses). This method, which combined family and within-family selection (Shaw et al., 1988), was expected to increase selection response for traits with low heritability (Falconer, 1981). Genotypes selected for intermediate expres-

Abbreviations: SSC, soluble solids content; TA, titratable acid content. 
sion were included as parents because they are expected to be in high frequency in the breeding population, and prediction of their cross performance was of operational significance. Twenty seedlings from each cross were planted in a randomized complete-block design, with a single plot of 10 plants in each of two blocks. After plant mortality and exclusion of nonflowering items, 301 and 285 seedlings were available for Set I and Set II, respectively.

Fruit were harvested from each seedling twice during Spring 1988. Seedlings in block 1 were sampled during the weeks beginning 25 Apr. and 16 May, and seedlings from block 2 during the weeks beginning 2 and 23 May, with $93 \%$ of the seedlings in each block successfully sampled on both dates. Block differences reflect the effects of both spatial variation and seasonal variation, because the average harvest date for seedlings in the second block was later in the season than for seedlings in the first. Fruit were stored, juice extracted, and SSC and TA determined as described by Shaw (1988). Individual samples included juice from at least three fruit, and under- or overripe fruit were discarded before juice extraction. In addition, each sample was assigned a subjective ripeness score before processing to further control between-sample variation for fruit ripeness. This score was generated by assigning a value of 1 to 5 to each individual fruit (with 5 indicating the ripest fruit) and averaging over all fruit included in the sample.

Means were calculated and analyses of variance (ANOVA) conducted for six categories of parental cross combination $(\mathrm{H}$ $\times \mathrm{H}, \mathrm{H} \times \mathrm{I}, \mathrm{H} \times \mathrm{L}, \mathrm{I} \times \mathrm{I}, \mathrm{I} \times \mathrm{L}, \mathrm{L} \times \mathrm{L}) \mathrm{using}$ individual plant means, i.e., individual seedling values averaged over two harvest dates. Category means were compared using a Bonferroni's adjusted $t$ test. Evaluation of cross categories using offspring values served as a progeny test for detecting differences in breeding value among the genotypes used as parents.

Genetic effects were further tested and variance components were estimated using 18 of the 20 crosses within each set. The two-crosses excluded from each set were included originally to increase the precision of category-mean comparisons, but did not contribute information to genetic analyses. For both sets, the 18 crosses resulted from a 3 x 6 factorial mating design (Comstock and Robinson, 1948), with 273 and 261 seedlings for Set I and Set II, respectively. Males (M), females (F), and $\mathrm{M} \times \mathrm{F}$ interaction were included in the analyses as genetic sources; blocks and harvests were included as environmental sources, with harvests treated as repeated measures. The subjective ripeness score was included as a covariate. All factorial interactions between genetic and environmental sources were included in ANOVAs conducted using SAS procedure GLM (SAS, 1985), but sums of squares and degrees of freedom for interactions that were nonsignificant in all analyses were pooled into a common error term. The resulting form and expected mean squares for these analyses are given in Table 1; the significance of main effects and interactions was tested using direct ratios or synthetic combinations (Milliken and Johnson, 1984) of these expected mean squares. Model components of variance were estimated using linear combinations of the expected mean squares in Table 1 (Searle, 1971); causal genetic components and heritabilities were estimated as follows (Hallauer and Miranda, 1981):

$$
\begin{aligned}
& \sigma_{\mathrm{A}}^{2}=2\left(\sigma_{\mathrm{M}}^{2}+\sigma_{\mathrm{F}}^{2}\right) \\
& \sigma_{\mathrm{D}}^{2}=4\left(\sigma_{\mathrm{MF}}^{2}\right)
\end{aligned}
$$

$$
\begin{gathered}
h^{2}=2\left(\sigma_{M}^{2}+\sigma_{F}^{2}\right) /\left(\sigma_{M}^{2}+\underset{\sigma_{F}}{\sigma_{F}^{2}+\sigma_{M F}^{2}+}\right. \\
\left.\sigma^{2}{ }_{P(B M F)}+\sigma^{2}{ }_{B H M F}+\sigma^{2}\right) \\
H^{2}=\left[2\left(\sigma_{M}^{2}+\sigma_{F}^{2}\right)+4\left(\sigma_{M F}^{2}\right)\right] /\left(\sigma_{M}^{2}+\sigma_{F}^{2}+\right. \\
\left.\sigma_{M F}^{2}+\sigma_{P(B M F)}^{2}+\sigma_{B H M F}^{2}+\sigma^{2}\right)
\end{gathered}
$$

In Eqs. [1] through [4], $\sigma^{2}{ }_{\mathrm{A}}$ and $\sigma^{2}$ are estimates of the additive and dominance genetic components of variance and $\mathrm{h}^{2}$ and $\mathrm{H}^{2}$ are the narrow- and broad-sense heritabilities on an individual plant basis.

The assumptions required for valid estimation of genetic parameters were discussed for quality traits by Shaw et al. (1987) and, in general, for strawberries by Shaw et al. (1988). As always, parameter estimates pertain to specific populations of genetic and environmental effects, and prediction of selection response outside the intended reference is invalid. An additional caution for this study is that parents were sampled at random from subpopulations created by divergent selection, possibly resulting in linkage disequilibrium. The presence of linkage disequilibrium is of minor concern when the objective is demonstration of selection response: differences in genetic parameters between selected and control populations are evidence of differentiation, regardless of origin. However, future selection responses predicted by use of parameter estimates from the pooled population will be inflated.

One consequence of divergent selection for SSC and TA, followed by intermating of selected genotypes, is that allele frequencies for the genes that condition these traits will shift toward intermediate values (Bulmer, 1980). Successful selection is expected to change genetic variances, and selection for intermediate allele frequencies can increase genetic variance if original frequencies were skewed towards fixation (Falconer, 1981; Sharloo, 1964). Changes in genetic variance resulting from divergent selection followed by intermating can be evaluated by comparing current estimates of genetic parameters for selected sets with parameters estimated in the original survey (Shaw et al., 1987), or by comparing current estimates for selected sets with those for unselected controls. For this study, Set I and Set II served as selected and control populations for $\mathrm{SSC}$, respectively, and the converse was true for TA. This treatment was valid because genotypes selected for SSC were chosen without consideration of TA phenotype, and selection for TA was performed without regard for SSC. The appropriate use of alternate sets as unselected controls also depends on independence of the genetic effects that condition SSC and TA, an assumption that was substantiated by the absence of significant genotypic correlations between these traits (Shaw, 1988).

\section{Results}

Highly significant differences $(P<0.01)$ were detected between cross-category means for SSC, and multiple comparison tests separated the six categories into three subgroups (Table 2). Progenies with two parents selected for high SSC had the highest mean SSC, and progenies with one high SSC parent had significantly higher means than those with only intermediate and low SSC parents. No differences were detected between $\mathrm{H}$ x $\mathrm{I}$ and $\mathrm{H} \times \mathrm{L}$ crosses, or among $\mathrm{I} \times \mathrm{I}, \mathrm{I} \times \mathrm{L}$, or $\mathrm{L} \times \mathrm{L}$ crosses. The mean for progenies in the central subgroup $(\mathrm{H} \mathrm{x}$ I, H x L) was slightly skewed toward the lower category, consistent with directional dominance for alleles that condition low SSC (Falconer, 1981).

ANOVA results and genetic parameter estimates for SSC 
Table 1. Form and expected mean squares (EMS) for ANOVA for SSC and TA in two factorial sets of crosses.

\begin{tabular}{|c|c|c|c|}
\hline \multirow[b]{2}{*}{ Source } & \multicolumn{2}{|c|}{$\begin{array}{c}\text { Degrees of } \\
\text { freedom }\end{array}$} & \multirow[b]{2}{*}{$\mathrm{EMS}^{\mathbf{z}}$} \\
\hline & Set I & Set II & \\
\hline Ripeness & 1 & 1 & $\cdot$ \\
\hline Block (B) & 1 & 1 & $\sigma^{2}+k_{1} \sigma_{\mathrm{BHFM}}^{2}+k_{2} \sigma_{\mathrm{P}(\mathrm{BFM})}^{2}+\mathrm{k}_{3} \sigma_{\mathrm{BH}}^{2}+k_{8} \sigma_{\mathrm{B}}^{2}$ \\
\hline Female $(F)$ & 2 & 2 & $\sigma^{2}+k_{1} \sigma_{B H F M}^{2}+k_{2} \sigma_{P(B F M)}^{2}+k_{4} \sigma_{F M}^{2}+k_{7} \sigma_{F}^{2}$ \\
\hline Male (M) & 5 & 5 & $\sigma^{2}+k_{1} \sigma_{B H F M}^{2}+k_{2} \sigma_{P(B F M)}^{2}+k_{4} \sigma_{F M}^{2}+k_{6} \sigma_{M}^{2}$ \\
\hline $\mathrm{F} \times \mathrm{M}$ & 10 & 10 & $\sigma^{2}+k_{1} \sigma^{2}{ }_{B H F M}+k_{2} \sigma_{P(B F M)}^{2}+k_{4} \sigma_{F M}^{2}$ \\
\hline $\mathrm{Plant} / \mathrm{B} \times \mathrm{F} \times \mathrm{M}$ & 272 & 260 & $\sigma^{2}+\mathrm{k}_{2} \sigma_{P(\mathrm{BFM})}^{2}$ \\
\hline Harvest $(\mathrm{H})$ & 1 & 1 & $\sigma^{2}+k_{1} \sigma_{B H F M}^{2}+k_{3} \sigma_{B H}^{2}+k_{5} \sigma_{H}^{2}$ \\
\hline $\mathrm{B} \times \mathrm{H}$ & 1 & 1 & $\sigma^{2}+k_{1} \sigma_{B H F M}^{2}+k_{3} \sigma_{B H}^{2}$ \\
\hline $\mathrm{B} \times \mathrm{H} \times \mathrm{F} \times \mathrm{M}$ & 10 & 6 & $\sigma^{2}+\mathrm{k}_{1} \sigma_{\text {BHFM }}^{2}$ \\
\hline Error & 272 & 228 & $\sigma^{2}$ \\
\hline
\end{tabular}

${ }^{z}$ Coefficients were estimated using SAS procedure GLM and Type III sum of squares as follows:

\begin{tabular}{lrr} 
& Set I & \multicolumn{1}{c}{ Set II } \\
\hline $\mathrm{k}_{1}$ & 7.2 & 7.1 \\
$\mathrm{k}_{2}$ & 1.8 & 1.7 \\
$\mathrm{k}_{3}$ & 126.5 & 90.5 \\
$\mathrm{k}_{4}$ & 28.9 & 21.7 \\
$\mathrm{k}_{5}$ & 245.3 & 181.0 \\
$\mathrm{k}_{6}$ & 85.7 & 63.6 \\
$\mathrm{k}_{7}$ & 166.8 & 113.4 \\
$\mathrm{k}_{8}$ & 252.9 & 149.5
\end{tabular}

Table 2. SSC for six cross classifications among parents selected for high $(\mathrm{H})$, intermediate $(\mathrm{I})$ and low $(\mathrm{L})$ expression.

\begin{tabular}{lccc}
\hline \hline $\begin{array}{l}\text { Cross } \\
\text { classification }\end{array}$ & $\begin{array}{c}\text { Crosses } \\
\text { (no.) }\end{array}$ & $\begin{array}{c}\text { Seedlings } \\
\text { (no.) }\end{array}$ & $\begin{array}{c}\text { SSC } \\
(\%)^{2}\end{array}$ \\
\hline $\mathrm{H} \times \mathrm{H}$ & 2 & 26 & $11.6 \mathrm{a}$ \\
$\mathrm{H} \times \mathrm{I}$ & 4 & 64 & $10.1 \mathrm{~b}$ \\
$\mathrm{H} \times \mathrm{L}$ & 4 & 53 & $10.4 \mathrm{~b}$ \\
$\mathrm{I} \times \mathrm{I}$ & 3 & 50 & $9.2 \mathrm{c}$ \\
$\mathrm{I} \times \mathrm{L}$ & 4 & 71 & $9.2 \mathrm{c}$ \\
$\mathrm{L} \times \mathrm{L}$ & 3 & 44 & $9.1 \mathrm{c}$ \\
\hline
\end{tabular}

${ }^{2}$ Values not followed by a common letter differ significantly $(P<0.05)$ compared with a Bonferroni's weighted multiple comparison test.

Table 3. Results of ANOVA for SSC using seedlings from factorial crosses among divergent selections (Set I) and controls (Set II).

\begin{tabular}{lccccc}
\hline \hline & \multicolumn{2}{c}{ Divergent } & & \multicolumn{2}{c}{ Controls } \\
\cline { 2 - 3 } \cline { 5 - 6 } selections & & & Mean & \\
Mean & & & & square & $\mathrm{F}$ \\
\hline S o u r c e & 30.7 & $8.5^{* *}$ & & 16.9 & $3.2^{*}$ \\
Ripeness & 287.0 & 1.6 & & 130.5 & $<1.0$ \\
Block (B) & 121.0 & $18.9^{* *}$ & & 68.9 & $4.3^{*}$ \\
Female (F) & 38.8 & $6.1^{*}$ & & 15.6 & $<1.0$ \\
Male (M) & 6.4 & 1.1 & & 15.9 & 1.2 \\
$\mathrm{~F} \times \mathrm{M}$ & 4.2 & 1.2 & & 6.0 & 1.1 \\
Plant $/ \mathrm{B} \times \mathrm{F} \times \mathrm{M}$ & 150.0 & $<1.0$ & & 82.8 & 1.0 \\
Harvest $(\mathrm{H})$ & 179.7 & $38.7^{* *}$ & & 151.5 & $13.4^{*}$ \\
$\mathrm{~B} \times \mathrm{H}$ & 4.8 & 1.3 & & 11.3 & $2.1^{*}$ \\
$\mathrm{~B} \times \mathrm{H} \times \mathrm{F} \times \mathrm{M}$ & 3.6 & & 5.3 & \\
Error & & &
\end{tabular}

","*n Significant at $P=0.05$ and 0.01 , respectively.

demonstrated a similar response to environmental sources of variation but different patterns of genetic variation for selected and control sets. Regression of SSC values on ripeness scores was significant for both selected and control sets (Table 3), but correction for ripeness as a covariate reduced the phenotypic variance by $<1 \%$ in both cases. Differences in the subjective ripeness score may reflect genetic variation for color and firmness that is independent of SSC. Block and harvest sources of variance were nonsignificant, and block $x$ harvest interaction variances were significant for both sets. Significant interactions, together with nonsignificant main effects, indicated that the environments defined by block and harvest classifications have a nonlinear effect on SSC. Variances due to males and females were significant for Set I, and the variance among females was significant for Set II. The additive genetic variance estimated for SSC using crosses among divergent parents was 2.3 times greater than for crosses among unselected controls $\left(\sigma_{\mathrm{A}}^{2}=2.14\right.$ vs. 0.96, Table 4), with corresponding differences in narrowsense heritability $\left(h^{2}=0.41\right.$ and 0.15$)$. No significant dominance variance was detected for either set.

Differences among cross-category means for TA were highly significant and were consistent with differences in breeding value among all three parental classes (Table 5). Progenies with two high and two low TA parents had the highest and lowest category means, respectively. Progenies with two intermediate parents had intermediate values that differed significantly from progeny means at both extremes. Means for $\mathrm{H} \times \mathrm{L}$ and $\mathrm{I} \times \mathrm{I}$ categories did not differ significantly, consistent with largely additive effects for the genes that condition TA.

The ANOVA results and variance component estimates for

Table 4. Genetic components of variance and heritabilities estimated for SSC using seedlings from factorial crosses among divergent selections and controls, with standard errors in parentheses.

\begin{tabular}{lcc}
\hline \hline Parameter & $\begin{array}{c}\text { Divergent } \\
\text { selections } \\
\text { (Set 1) }\end{array}$ & $\begin{array}{c}\text { Controls } \\
\text { (Set 11) }\end{array}$ \\
\hline$\sigma^{2}{ }_{\mathrm{A}}$ & $2.14(1.10)$ & $0.96(0.93)$ \\
$\mathrm{\sigma}^{2}{ }_{\mathrm{D}}$ & $0.12(0.35)$ & $0.72(1.25)$ \\
$\mathrm{h}^{2}$ & $0.41(0.15)$ & $0.15(0.14)$ \\
$\mathrm{H}^{2}$ & $0.44(0.22)$ & $0.26(0.23)$ \\
\hline
\end{tabular}


Table 5. TA for six cross classifications among parents selected for high $(\mathrm{H})$, intermediate $(\mathrm{I})$, and low $(\mathrm{L})$ expression.

\begin{tabular}{lccc}
\hline \hline $\begin{array}{l}\text { Cross } \\
\text { classification }\end{array}$ & $\begin{array}{c}\text { Crosses } \\
\text { (no.) }\end{array}$ & $\begin{array}{c}\text { Seedlings } \\
\text { (no.) }\end{array}$ & $\begin{array}{c}\text { TA } \\
(\%)\end{array}$ \\
\hline $\mathrm{H} \times \mathrm{H}$ & 1 & 15 & $1.15 \mathrm{a}$ \\
$\mathrm{H} \times \mathrm{I}$ & 3 & 33 & $0.98 \mathrm{~b}$ \\
$\mathrm{H} \times \mathrm{L}$ & 4 & 44 & $0.87 \mathrm{c}$ \\
$\mathrm{I} \times \mathrm{I}$ & 2 & 18 & $0.84 \mathrm{c}$ \\
$\mathrm{I} \times \mathrm{L}$ & 5 & 67 & $0.78 \mathrm{~cd}$ \\
$\mathrm{~L} \times \mathrm{L}$ & 5 & 70 & $0.75 \mathrm{~d}$ \\
\hline
\end{tabular}

${ }^{2}$ Values not followed by a common letter differ significantly $(P<0.05)$ compared with a Bonferroni's weighted multiple comparison test.

Table 6. Results of ANOVA for TA using seedlings from factorial crosses among divergent selections (Set II) and controls (Set I).

\begin{tabular}{lccccc}
\hline \hline & \multicolumn{2}{c}{$\begin{array}{c}\text { Divergent } \\
\text { selections }\end{array}$} & & \multicolumn{2}{c}{ Controls } \\
\cline { 2 - 3 } \cline { 5 - 6 } & $\begin{array}{c}\text { Mean } \\
\text { square }\end{array}$ & $\mathrm{F}$ & & $\begin{array}{c}\text { Mean } \\
\text { square }\end{array}$ & $\mathrm{F}$ \\
Source & 0.1 & $<1.0$ & 1.2 & 1.3 \\
Ripeness & 65.3 & $32.7^{* *}$ & & 191.4 & $146.2^{* *}$ \\
Block (B) & 55.1 & $7.3^{*}$ & & 1.0 & $<1.0$ \\
Female (F) & 58.8 & $7.8^{* *}$ & & 6.6 & 1.7 \\
Male (M) & 7.5 & $2.2^{*}$ & & 3.8 & 1.5 \\
$\mathrm{~F} \times \mathrm{M}$ & 3.4 & $1.7^{* *}$ & & 1.8 & $2.0^{* *}$ \\
Plant/B $\times \mathrm{F} \times \mathrm{M}$ & 71.1 & $35.6^{* *}$ & & 100.1 & $76.0^{* *}$ \\
Harvest (H) & 0.1 & $<1.0$ & & 0.7 & $<1.0$ \\
$\mathrm{~B} \times \mathrm{H}$ & 1.0 & $<1.0$ & & 1.3 & 1.4 \\
$\mathrm{~B} \times \mathrm{H} \times \mathrm{F} \times \mathrm{M}$ & 2.0 & & & 0.9 & \\
Error & & &
\end{tabular}

*,**Significant at $P=0.05$ and 0.01 , respectively.

Table 7. Genetic components of variance and heritabilities estimated for TA using seedlings from factorial crosses among divergent selections and controls, with standard errors in parentheses.

\begin{tabular}{|c|c|c|}
\hline Parameter & $\begin{array}{c}\text { Divergent } \\
\text { selections } \\
\text { (Set II) }\end{array}$ & $\begin{array}{c}\text { Controls } \\
\text { (Set I) }\end{array}$ \\
\hline$\sigma_{\mathrm{A}}^{2}$ & $2.42(1.10)$ & $0.26(0.09)$ \\
\hline$\sigma_{\mathrm{D}}^{2}$ & $0.76(0.57)$ & $0.24(0.24)$ \\
\hline$h^{2}$ & $0.57(0.26)$ & $0.17(0.06)$ \\
\hline $\mathrm{H}^{2}$ & $0.76(0.30)$ & $0.30(0.14)$ \\
\hline
\end{tabular}

TA in selected and control sets demonstrated similar response to environmental sources and different patterns of genetic variance. Regression of TA on ripeness score was nonsignificant for both selected and unselected sets (Table 6). Variances due to the main effects of blocks and harvests were significant, and block $\mathrm{x}$ harvest interactions were nonsignificant for both sets. These results contrasted with those described above for SSC and suggest that the traits respond differently to the environments defined by block and harvest sources of variance. Variances due to males, females, and $\mathrm{M} \times \mathrm{F}$ interactions were significant for Set II, whereas none of these genetic variances were significant for Set I. The additive genetic variance for TA, estimated using crosses among divergent parents, was 9.2 times larger than for the unselected controls $\left(\sigma_{\mathrm{A}}^{2}=2.42\right.$ and 0.26 , Table 7).

\section{Discussion}

The significant differences detected among offspring values from different cross categories demonstrate response to selection for breeding value. For SSC, selection response was detected in the upward direction, but selection was ineffective in the downward direction. Response to selection for TA was demonstrated in both upward and downward directions.

Divergent selection followed by intermating also generated populations that express larger total genetic variances than crosses among unselected genotypes. Genetic parameters estimated for SSC in Set I also differed from those obtained in the initial survey, where dominance effects were significant, and explained the major fraction of the genetic variance. However, the genetic parameters estimated for SSC using unselected parents (Set II) were similar to those estimated previously (Shaw et al., 1987). Heritabilities estimated for TA in Set II were larger than those estimated in the original survey, whereas heritabilities for TA in the control set were smaller.

The magnitude and distribution of genetic variance components depend on allele frequencies for genes that condition trait values, and selection response for quantitative characters is realized by changing allele frequencies. Heritabilities can increase or decrease with successful selection, depending on initial allele frequencies and the mode of gene action (Falconer, 1981). Although substantial increases in genetic variances were observed, caution in predicting future selection response from these current estimates is needed. As discussed previously, pooling of subpopulations created by divergent selection can generate linkage disequilibrium, which can inflate estimates of genetic variance.

The demonstration of selection response for breeding value contrasts with results reported previously for genotypic selection of SSC, where no response was detected (Shaw, 1988). Phenotypic values for selections in the original seedling test (Shaw et al., 1987), selection means in clonal tests (Shaw, 1988), and current offspring means were compared to discern between potential alternative explanations for this discrepancy. The product-moment correlation between original seedling values and clonal selection means for SSC was nonsignificant $(r=0.06)$, whereas original values and current offspring means for the same selections were positively correlated $(r=0.64, P<0.06)$. These observations suggest that the failure to obtain clonal selection response for SSC resulted from the instability of genetic effects over differing locations or propagation systems, i.e., genotype $\times$ environment interaction, rather than a lack of adequate genetic variation. The increase in additive genetic variance observed for crosses among divergent SSC selections relative to unselected controls is consistent with substantial changes in allele frequency towards intermediacy, with alleles that confer high SSC partially recessive and infrequent in the base population (Falconer, 1981). Successful selection for SSC in seedlings suggests that improvement within this germplasm base may be possible for vegetatively propagated cultivars, provided that evaluations are performed using test criteria that simulate commercial conditions. However, selection response for SSC under commercial conditions will depend on the expression of genetic differences in production environments that induce heavy demands on available carbohydrates and has yet to be demonstrated.

The response to selection demonstrated for TA using offspring means was consistent with results obtained previously for clonal selection response (Shaw, 1988). Product-moment correlations between phenotypic values in the original seedling test and selection means in the clonal trials were significant and large $(r=0.78)$, as were correlations between seedling phenotypic value and current offspring means for the same selections $(r=0.91)$. Genetic effects that condition TA were stable to the environmental conditions and cultural systems that dif- 
fered between seedling and clonal tests. Within the current germplasm base, selection strategies that use genetic differences for expression of TA to control strawberry flavor are expected to be reliable.

\section{Literature Cited}

Bulmer, M.G. 1980. The mathematical theory of quantitative genetics. Oxford University Press, New York.

Comstock, R.E. and H.F. Robinson. 1948. The components of genetic variation in populations of biparental progenies and their use in estimating the average degree of dominance. Biometrics 4:254-266.

Falconer, D.S. 1981. Introduction to quantitative genetics. 2nd ed. Longman Group, London.

Hallauer, A. R., and J.B. Miranda. 1981. Quantitative genetics in maize breeding.. 2nd ed. Iowa State University Press, Ames.

Milliken, G. A., and D.E. Johnson. 1984. Analysis of messy data. vol. I: Designed experiments. Van Norstrand Reinhold, New York.
SAS Institute, Inc. 1985. SAS user's guide: Statistics. version 5. SAS Institute, Inc., Cary, N.C.

Searle, S.R. 1971. Linear models. Wiley, New York.

Sharloo, W. 1964. The effect of disruptive and stabilizing selection on the expression of cubitus interrupts in Drosophila. Genetics 50:553562.

Shaw, D.V. 1988. Genotypic variation and genotypic correlations for sugars and organic acids in strawberries. J. Amer. Soc. Hort. Sci. 113:770-774.

Shaw, D. V., R.S. Bringhurst, and V. Voth. 1987. Genetic variation for quality traits in an advanced cycle breeding population of strawberries. J. Amer. Soc. Hort. Sci. 112:699-702.

Shaw, D. V., R. S. Bringhurst, and V. Voth. 1988.. Quantitative genetic variation for resistance to leaf spot in California strawberries. J. Amer. Soc. Hort. Sci. 113:451-456.

Sweeny, J. P., V.J. Chapman, and P.A. Hepner, 1970. Sugar, acid, and flavor in fresh fruit. J. Amer. Dietetic Assn. 57:432-435. 